University of Wollongong

Research Online

Faculty of Engineering and Information

Faculty of Engineering and Information

Sciences - Papers: Part A

Sciences

$1-1-2014$

\title{
Feasibility study on the implementation of IEEE 802.11 on cloud-based radio over fibre architecture
}

Sen Zhang

University of Wollongong, sz765@uowmail.edu.au

Daniel Franklin

University of Wollongong, University of Technology Sydney, danielf@uow.edu.au

Follow this and additional works at: https://ro.uow.edu.au/eispapers

Part of the Engineering Commons, and the Science and Technology Studies Commons

Research Online is the open access institutional repository for the University of Wollongong. For further information contact the UOW Library: research-pubs@uow.edu.au 


\title{
Feasibility study on the implementation of IEEE 802.11 on cloud-based radio over fibre architecture
}

\author{
Abstract \\ This paper investigates the throughput performance of the IEEE 802.11 MAC when the physical layer is \\ implemented remotely on a cloud-based SDR platform. An analytical model which considers a non-zero \\ late ACK arrival probability is proposed to analyse throughput performance. Both conventional DCF and \\ the Block ACK enhancement from current IEEE 802.11 standards are analysed using the proposed model. \\ Results show that the network delay variance significantly degrades the performance of conventional DCF \\ while enabling Block ACK significantly reduces this degradation.

\section{Keywords} \\ cloud, study, radio, over, fibre, architecture, implementation, ieee, 802,11 , feasibility \\ Disciplines \\ Engineering | Science and Technology Studies

\section{Publication Details} \\ S. Zhang \& D. Franklin, "Feasibility study on the implementation of IEEE 802.11 on cloud-based radio over \\ fibre architecture," in IEEE International Conference on Communications (ICC), 2014, pp. 2891-2896.
}




\title{
Feasibility Study on the Implementation of IEEE 802.11 on Cloud-Based Radio over Fibre Architecture
}

\author{
Sen Zhang* and Daniel R. Franklin ${ }^{\dagger}$ \\ ${ }^{*}$ School of Electrical Computer and Telecommunications Engineering \\ Engineering and Information Sciences, University of Wollongong, Australia \\ ${ }^{\dagger}$ School of Computing and Communications, Faculty of Engineering \& IT \\ University of Technology, Sydney, Australia \\ Email: sz765@uowmail.edu.au, daniel.franklin@uts.edu.au
}

\begin{abstract}
This paper investigates the throughput performance of the IEEE 802.11 MAC when the physical layer is implemented remotely on a cloud-based SDR platform. An analytical model which considers a non-zero late ACK arrival probability is proposed to analyse throughput performance. Both conventional DCF and the Block ACK enhancement from current IEEE 802.11 standards are analysed using the proposed model. Results show that the network delay variance significantly degrades the performance of conventional DCF while enabling Block ACK significantly reduces this degradation.
\end{abstract}

\section{INTRODUCTION}

Cloud-based remote implementation of physical layer signal processing and MAC protocols is an emerging field with substantial commercial interest [1], [2]. By moving most components of a wireless transceiver into the cloud, enormous opportunities for reductions in hardware and maintenance costs can be created, while unleashing the enormous power of software-defined radio (SDR) in a remarkably flexible and scalable manner. Despite these benefits, as yet few studies have been conducted into the use of a general-purpose cloud computing platform for such an architecture, due to the inherent challenges of variable processing and network latency. While these challenges are substantial for many applications, there are a number of specific protocols and radio standards for which a general-purpose cloud implementation is practically feasible, albeit with a performance penalty or other limitations.

A key step towards such systems is the Radio-over-Fibre (RoF) architecture, in which the baseband part of the physical layer and higher-layer protocols are implemented on a remote centralised processor, separated from the rest of the physical radio hardware by a single-hop optical link [3], [4]. Baseband waveform signals are generated in-cloud by a software-defined transmitter and transported over the fibre (typically via Ethernet encapsulation) to a relatively simple generic transceiver front-end; received signals are similarly conveyed to the software-defined receiver in the cloud and demodulated. The addition of the fibre link introduces a small delay compared to a conventional software-defined radio transceiver in which there is a negligible and constant latency between the processor and transceiver front-end.

RoF has been evaluated with a number of existing wireless communications technologies, including IEEE 802.11 (WiFi).
However, most existing research into wireless performance over RoF is limited by the need to place the processing infrastructure relatively close to the physical part of the radio. This is because most existing short-range wireless protocols that might be implemented on RoF have strict timing constraints: for example, IEEE 802.11 specifies the maximum propagation delay to be $1 \mu \mathrm{s}$. Therefore, when additional latencies are introduced by the need to transport baseband signals across a network between the SDR and physical transceiver components, the performance of such protocols may be heavily degraded. To extend the RoF paradigm to a general-purpose cloud-based processing centre, analytical models need to consider the latency and jitter introduced by transporting each waveformbearing data frame through multiple routers and switches.

The IEEE 802.11 MAC protocol schedules packet transmission using the Distributed Coordination Function (DCF), which uses a stop-and-wait ARQ scheme for data recovery at the MAC level. However, although it is an effective mechanism for dealing with packets lost due to transmission errors, the throughput performance of traditional DCF is limited by the need to wait for acknowledgements before sending the next frame. Xiao et al. suggests that due the overhead of DCF, there is always a limit to DCF throughput regardless of the bit rate [5].

When DCF is implemented on a RoF system, additional delays caused by variable network latency lead to frequent transmission failure, which greatly reduces throughput. The IEEE $802.11 \mathrm{e} / \mathrm{n}$ protocol enhancements introduce a block acknowledgement scheme (Block ACK) to reduce the overhead introduced by needing to wait for acknowledgement of every unicast frame. The basic concept of the Block ACK mechanism is that as soon as one station (STA) successfully obtains a transmission opportunity, it can transmit a series of MPDUs with an interval of one Short Interframe Space (SIFS). Instead of waiting for an ACK for each individual MPDU, one Block ACK aggregating all ACKs for the previous MPDUs received by the receiver will be returned to the transmitter for retransmission decision-making.

The performance of the basic DCF mechanism over a onehop RoF system is analysed by Kalantari et al. [6]. The authors prove that the RoF fibre length over which DCF will correctly operate is limited due to the value of $A C K \_$timeout. Each 
kilometre of fibre adds an additional deterministic latency of approximately $5 \mu \mathrm{s}$. If the propagation delay on the fibre exceeds $A C K$ timeout, every frame will time out, and the transmitter will keep retransmitting the same frame until it is discarded due to retry limit being reached. At the receiver side, the receiver will ignore duplicated frames, although they are likely to be received without error. Hence, the throughput drops abruptly to zero when this threshold is reached.

Possible solutions to overcoming the fibre length constraint include increasing $A C K \_$timeout value [7], modifying the slottime value [8], and using frame aggregation with the Block ACK mechanism discussed previously [9]. Although increasing $A C K \_$timeout can improve system performance to some extent, this improvement implicitly assumes a deterministic network latency, which is valid for RoF but not for multihop networks in general [7]. It is also not practical to increase $A C K_{-}$timeout for upstream transmissions from legacy devices to RoF DCF implementations, limiting the benefits to one direction only in this case. Modifying slottime is impractical as it requires all participating stations to be modified. Deronne et al. demonstrate improved performance through simulations when frame aggregation and Block ACK mechanism are enabled in a RoF DCF implementation; however, a general analytical model is not provided [9].

The model proposed in this paper is applicable for IEEE 802.11 implementations over cloud-based architectures such as [1], [10], [11], [12]. Variable latencies are introduced by queueing process in switches and routers in a multihop network. Hence, realistic statistical model should be used to represent network latency. According to the experimental results in [13], it is suggested that long-tailed distributions such as the Weibull distribution or shifted Gamma distribution are suitable for modelling network latency distributions. In this paper, we have adopted the shifted Gamma distribution. To the best of our knowledge, this is the first attempt to mathematically analyse throughput performance of both basic DCF and Block ACK mechanism of IEEE 802.11 when implemented on a cloud-like architecture that considers realistic network characteristics.

This paper is organized as follows: Section II-A briefly describes the basic DCF mechanism in IEEE 802.11; Section II-B describes the Block ACK technique; Section III describes the proposed analytical models of both basic DCF and DCF with Block ACK with statistically distributed latency introduced by a multihop network; Section IV presents and discusses some numerical results based on the proposed analytical model; finally, Section V provided the conclusions of this paper.

\section{IEEE 802.11 ACCESS SCHEMES}

\section{A. DCF Scheme}

There are two access mechanisms in IEEE 802.11's DCF. The first is a basic two-way handshaking mechanism (data/ACK), while the second uses a four-way handshaking mechanism (RTS/CTS/data/ACK) for channel reservation. In this paper, only the basic mechanism will be discussed.

Basic DCF relies on channel sensing and backoff mechanisms to reduce collision probability. The basic protocol is a variant of stop-and-wait ARQ; the receiver is required to return an ACK to the sender after each frame is successfully

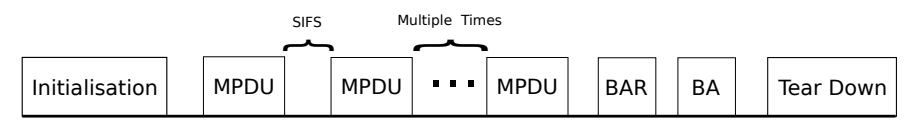

Fig. 1. Block ACK procedure

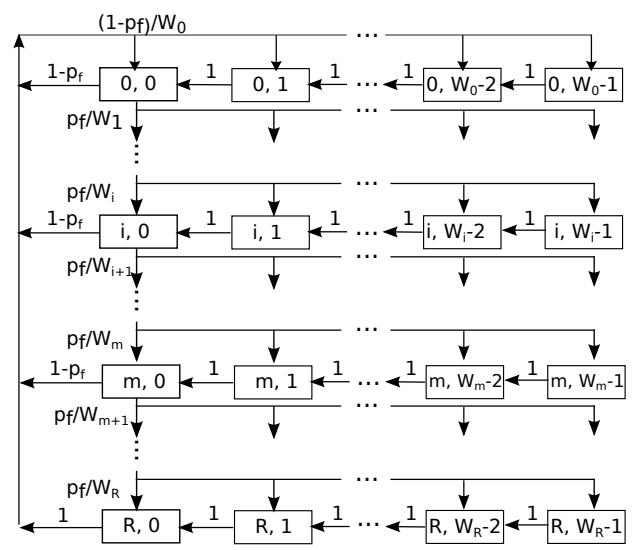

Fig. 2. Markov chain model for DCF backoff procedure

decoded before the sender transmits the next frame, subject to exponential backoff resulting from contention between multiple stations. Basic DCF has been extensively studied for many years; the protocol details of basic DCF and analyses of its fundamental performance limitations are discussed extensively in [14], [15], [5].

\section{B. Block ACK Scheme}

The Block ACK process uses an aggregated acknowledgement frame containing all the ACKs of requested frames in order to reduce the overhead caused by the stop-and-wait ARQ mechanism in conventional DCF. Specifically, as shown in Fig. 1, BA process includes four stages: 1) initialisation with exchange of $A D D B A$ request (add Block ACK Request) and $A D D B A$ Response (add Block ACK response); 2) data transmission with multiple MPDUs separated by SIFS; 3) Block ACK returns as requested by Block ACK Request (BAR) from transmitter; 4) Block tear-down stage with exchange of DELBA (delete Block ACK) frames. Because data transmission happens at stage 2 and 3, only these two stages will be analysed for throughput analysis in this paper.

The Block ACK mechanism allows multiple MPDUs to be transmitted back-to-back with with an inter-frame interval of one SIFS period without further channel contention. The channel is contended only once by either basic DCF-based head-of-burst (HOB) or RTS/CTS mechanisms. After each data block is transmitted, a BA is returned from receiver to sender on request (via frame BAR). BARs are retransmitted if there is no response to a BAR within the timeout period. The BA lists missing MPDUs in a $64 \times 16$-bit bitmap, and these will then be retransmitted in a subsequent burst by the transmitter once it has received the BA.

\section{ANALYTICAL Model}

\section{A. Markov Chain Model}

The bi-dimensional Markov chain model initially proposed by Bianchi in [15] for modelling backoff behaviour in IEEE 
802.11 under a saturation scenario is now well-known and widely used throughout the literature. Saturation is defined as a state in which every station in the system always has one or more packets ready to be transmitted. In [15], packets subject to collisions can be retransmitted an unlimited number of times until it is received successfully. However, the actual IEEE 802.11 standard [14] stipulates a maximum number of retry attempts. Bianchi's work has thus been extended by $\mathrm{Wu}$ et al. to consider the effects of finite retransmission limits [16].

The backoff process is modelled using a bi-dimensional Markov chain $\{s(t), b(t)\}$, where $s(t)$ represents the backoff stage while $b(t)$ represents the state of backoff counter.

For one STA, when transition probability is expressed as $P\{i, k \mid j, l\}^{1}$, unsuccessful transmission probability is $p_{f}$, transmission probability is denoted as $\tau, m$ represents the maximum backoff stage, $R$ stands for retry limit and $W_{i}$ is short for $C W_{i}$, then according to the backoff mechanism description in Section II-A, a set of non-null one-step transitions in this bi-dimensional Markov Chain can be obtained:

$$
\begin{cases}P\{i, k \mid i, k+1\}=1, & 0 \leq i \leq m, 0 \leq k \leq W_{i}-2 \\ P\{0, k \mid i, 0\}=\left(1-p_{f}\right) / W_{0}, & 0 \leq i \leq m-1,0 \leq k \leq W_{0}-1 \\ P\{i, k \mid i-1,0\}=p_{f} / W_{i}, & 1 \leq i \leq R, 0 \leq k \leq W_{i}-1 \\ P\{0, k \mid R, 0\}=1, & 0 \leq k \leq W_{0}-1\end{cases}
$$

The stationary probability is $b_{i, k}$, then we have:

$$
b_{i, 0}=p_{f}^{i} b_{0,0}, \quad 0<i<m
$$

According to [17], when considering the factor of retry limit, $b_{0,0}$ is:

$$
b_{0,0}= \begin{cases}\frac{2\left(1-2 p_{f}\right)\left(1-p_{f}\right)}{\left.W_{0}\left(1-p_{f}\right)\left(1-\left(2 p_{f}\right)^{R+1}\right)\right)+\left(1-2 p_{f}()\left(1-p_{f}^{R+1}\right)\right.}, & R \leq m \\ \frac{2\left(1-2 p_{f}\right)\left(1-p_{f}\right)}{Z}, & R>m\end{cases}
$$

where $Z$ is:

$$
\begin{aligned}
Z= & W_{0}\left(1-\left(2 p_{f}\right)^{m+1}\right)\left(1-p_{f}\right)+\left(1-p_{f}^{R+1}\right)\left(1-2 p_{f}\right) \\
& +2^{m} W_{0}\left(1-p_{f}^{R-m}\right)\left(1-2 p_{f}\right)
\end{aligned}
$$

Transmission probability is the sum of probabilities when Backoff Counter is zero at all stages:

$$
\tau=\sum_{i=0}^{R} b_{i, 0}=b_{0,0} \sum_{i=0}^{R} p_{f}^{i}=b_{0,0} \frac{1-p_{f}^{R+1}}{1-p_{f}}
$$

Having the value of $b_{0,0}$, it is easy to obtain $\tau$ as:

$$
\tau= \begin{cases}\frac{2\left(1-2 p_{f}\right)\left(1-p_{f}^{R+1}\right)}{\left.W_{0}\left(1-p_{f}\right)\left(1-\left(2 p_{f}\right)^{R+1}\right)\right)+\left(1-2 p_{f}\right)\left(1-p_{f}^{R+1}\right)}, & R \leq m \\ \frac{2\left(1-2 p_{f}\right)\left(1-p_{f}^{R+1}\right)}{Z}, & R>m\end{cases}
$$

In this paper, there are two types of failure: collisions and late ACK arrival (due to transport network latency between cloud and physical transceiver). Therefore, if the multiple station collision probability is $p_{c}$, then the total collision probability $p_{f}$ can be expressed as Eq. 7:

$$
p_{f}=1-\left(1-p_{c}\right)\left(1-p_{d}\right)=p_{c}+p_{d}-p_{c} p_{d}
$$

The multiple STAs collision probability may now be obtained:

$$
p_{c}=1-(1-\tau)^{n-1}
$$

\footnotetext{
${ }^{1} P\{i, k \mid j, l\}=P\{s(t+1)=i, b(t+1)=k \mid s(t)=j, b(t)=l\}$
}

$p_{d}$ depends on the probability distribution of round trip latency over network. Eq. 6 and Eq. 8 are nonlinear equations and can be solved numerically.

\section{B. DCF Saturation Throughput}

Throughput is defined as the data rate for successfully transmitted data (expressed in bit/sec). If $E\left[L_{P l d}\right]$ is denoted for the average size of successfully transmitted data and $E[T]$ represents average length of slot duration, then throughput $S$ can be expressed as:

$$
S=\frac{E\left[L_{P l d}\right]}{E[T]}
$$

There are four cases for DCF implementations operating on remote cloud-like systems: 1) idle - there are no station transmitting; 2) success - the channel is sensed busy because there is only one STA is transmitting and this STA receives an ACK within the ACK_timeout period; 3) Collision the channel is sensed busy because more than one STA is attempting to transmit at the same time; 4) Delay - channel is sensed busy when there is only one STA transmitting but this STA does not receive an ACK within the period of ACK_timeout.

To analyse throughput $S$, all possible durations and probabilities for one randomly chosen time slot should be investigated. We first analyse the time duration in four cases.

- $\quad T_{I}$ is denoted as the duration of the idle state; therefore the value of $T_{I}$ is the system slot time $\sigma$.

- For successful transmission, the duration time $T_{S}$ is the sum of data transmission time, SIFS, ACK transmission time and DIFS. Data is transmitted in units of MAC protocol data units (MPDUs). The time used to transmit each MPDU includes the PHY preamble transmission time $T_{P H Y_{h d r}}$ (at lowest control rate), and transmission time for the MPDU $T_{M P D U}$ at the data rate. $T_{M P D U}$ includes the data payload and MAC layer header and trailer, so $T_{M P D U}=T_{M A C_{h d r}}+$ $T_{\text {data }}$.

- When the transmitted data undergoes a collision with another transmitter, the transmitter and other STAs will defer their transmission for one Extended Interframe Space (EIFS) period. Therefore, the time duration in this situation $T_{C}$ is the sum of data transmission time and EIFS, where

$$
T_{E I F S}=T_{S I F S}+T_{P H Y_{h d r}}+T_{A C K}+\delta+T_{D I F S}
$$

- For the situation where transmission is successful but the ACK returns late due to transport network latency (cloud-transceiver communications delays), the transmitter cannot distinguish between the late ACK and a collision. This is because the results are same: the ACK does not return in time and a retransmission will therefore be initiated. Therefore, the duration time $T_{D}$ will be the same as $T_{C}$. Some literature calculates $T_{C}$ as the sum of data transmission time, $A C K_{-}$timeout and DIFS. However, because

$$
T_{A C K_{-} \text {timeout }}=T_{S I F S}+T_{P H Y_{h d r}}+T_{A C K}+\delta
$$


The expression using EIFS or ACK_timeout turn out to be same.

In summary, the time durations for the four cases are listed below:

$$
\left\{\begin{aligned}
T_{I}= & \sigma \\
T_{S}= & 2\left(T_{P H Y_{h d r}}+\delta\right)+T_{M P D U}+T_{\text {uplink }}+T_{\text {downlink }}+ \\
& T_{S I F S}+T_{A C K}+T_{D I F S} \\
T_{C}= & T_{P H Y_{h d r}}+T_{M P D U}+\delta+T_{A C K_{-} \text {timeout }}+T_{D I F S} \\
T_{D}= & T_{C}
\end{aligned}\right.
$$

where for simplification, round-trip time $(R T T)$ over the high speed network will replace $\left(T_{\text {uplink }}+T_{\text {downlink }}\right)$. Practically, $R T T$ not only includes the duration of both uplink and downlink transmission, but also the processing latency of the cloud-based SDR. Therefore, due to the variable processing and network latency in a real network and cloud, RTT now includes a random component. $E[R T T]$ indicates the mean value of $R T T$.

If the transmission probability of one STA is $\tau$ and there are $n$ STAs in the system, then channel idle probability $P_{I}$ is $(1-\tau)^{n}$, the probability with only one STA is $n(1-\tau)^{n-1}$, and the collision probability $P_{C}$ with more than one STAs transmitting at same time is $1-(1-\tau)^{n}-n(1-\tau)^{n-1}$. If the probability for the ACK not returning before $A C K_{-}$timeout expires is denoted $p_{d}$, the probabilities of the system for four cases is as follows:

$$
\left\{\begin{array}{l}
P_{I}=(1-\tau)^{n} \\
P_{S}=n \tau(1-\tau)^{n-1}\left(1-p_{d}\right) \\
P_{C}=1-(1-\tau)^{n}-n \tau(1-\tau)^{n-1} \\
P_{D}=n \tau(1-\tau)^{n-1} p_{d}
\end{array}\right.
$$

So Eq. 9 can be expressed as:

$$
S=\frac{P_{S} L_{P l d}}{P_{I} T_{I}+P_{S} T_{S}+P_{C} T_{C}+P_{D} T_{D}}
$$

\section{Block ACK Scheme Saturation Throughput}

This paper only investigates the HOB stage and data burst transmission stage in the Block ACK procedure. Because data burst transmission only occurs after the HOB and its ACK are successfully exchanged, the possible states with only HOBs participating are same as for the DCF scenario: idle, collision, successful transmission with late ACK delay. Two new cases which must be analysed under the condition of HOB success: data block with Block ACK frame returned; and data block transmission without Block ACK frame returned.

According to the standard, if the BA is not received, then another BAR will be retransmitted. The retry limit for Block ACK Requests is denoted as $R^{*}$. Because the timeout value of $\mathrm{BA}$ is different from $A C K_{\_}$timeout, the probability of the late arrival of a BA is denoted as $p_{d^{*}}$. The time duration for each case is analysed as follows:

- The idle situation remains the same as for plain DCF: $T_{I}=\delta$;

- In the HOB collision scenario, the time duration is $T_{C}$ which is same as the collision scenario $T_{C}$ in DCF;
- In HOB with late ACK delay scenario, the time duration is $T_{D_{-} A C K}$ which is same as delay scenario $T_{D}$ in DCF;

- In the case that the entire transmitted data block is received successfully and $\mathrm{BA}$ is returned within the timeout period, the total time $T_{S \_B l k}$ includes the time of $\mathrm{HOB}$ and ACK $\left(O_{H O B}\right)$, the time of remaining MPDUs $\left(T_{B l k}\right)$, the time of BAR which may be retransmitted multiple times $\left(O_{M B A R}\right)$ and the time of $\mathrm{BA}\left(O_{B A}\right)$.

$T_{S_{-} B l k}(i)=O_{H O B}+T_{B l k}+O_{M B A R}(i)+O_{B A}+D I F S$

where

$$
\left\{\begin{array}{l}
O_{M B A R}(i)=(i+1) O_{B A R} \\
O_{B A R}=T_{P H Y_{h d r}}+T_{B A R}+\delta+T_{B A_{-} \text {timeout }} \\
T_{B A_{-} t \text { timeout }}=T_{S I F S}+T_{P H Y_{h d r}}+T_{B A}+\delta \\
O_{B A}=T_{P H Y_{h d r}}+T_{B A}+\delta+E[R T T] / 2 \\
T_{B l k}=(N-1)\left(T_{P H Y_{h d r}}+T_{M P D U}+S I F S+\delta\right) \\
\quad+E[R T T] / 2 \\
O_{H O B}=2\left(T_{P H Y_{h d r}}+\delta\right)+T_{M P D U}+T_{S I F S} \\
\quad+T_{A C K}+E[R T T]
\end{array}\right.
$$

- If the retry limit is reached, there is no BA response to the BAR or the arrival of BA is later than the expiry of $B A_{-}$timeout, then the time for this case $T_{D \_B A}$ is the sum of the time of HOB and ACK $\left(O_{H O B}\right)$ and $R^{*}$ times of BAR retransmission and timeout.

$$
T_{D_{-} B A}=O_{H O B}+T_{B l k}+O_{B A R}\left(R^{*}\right)+T_{D I F S}
$$

In summary, the time durations for all cases are given by:

$$
\left\{\begin{array}{l}
T_{I}=\sigma \\
T_{S \_B l k}(i)=O_{H O B}+T_{B l k}+O_{M B A R}(i)+O_{B A}+D I F S \\
T_{C}=T_{P H Y_{h d r}}+T_{M P D U}+\delta+T_{A C K_{-} t i m e o u t}+T_{D I F S} \\
T_{D \_A C K}=T_{C} \\
T_{D \_B A}=O_{H O B}+T_{B l k}+O_{M B A R}\left(R^{*}\right)+T_{D I F S}
\end{array}\right.
$$

Because the scenarios of idle $\left(P_{I}\right)$, HOB collision $\left(P_{C}\right)$ and HOB with delayed ACK $\left(P_{D \_} A C K\right)$ are same as those in basic DCF, the probabilities for being in these states are the same as $P_{I}, P_{C}$ and $P_{D}$ from Eq. 13 respectively. There are two reasons for the difference between the probability of BA and ACK: firstly, the size of BA and ACK frames are different - the $\mathrm{BA}$ frame is much larger than an $\mathrm{ACK}$; and secondly, the BA frame is transmitted at the normal data transmission rate while ACKs are transmitted at the control rate, which is usually the lowest rate supported by the protocol. If the probability of late $\mathrm{BA}$ is denoted as $p_{b}^{*}$ and retry limit for BAR is $R^{*}$, then the probability of successful block transmission with successful BA return will be $P_{S_{-} B l k}=P_{S_{-} H O B}\left(\sum_{i=0}^{R^{*}} p_{d^{*}}^{i}\left(1-p_{d}^{*}\right)\right)$, where $P_{S_{-} H O B}$ represents the probability for successful HOB (identical to $P_{S}$ in Eq. 13). When the retry limit is reached but there is no response received by originator, the all MPDUs except for the HOB are considered to have failed. In this failure case, the state probability is $P_{D_{-} B A}=P_{S_{-} H O B} p_{d^{*}}^{R^{*}+1}$. 
In summary, the probabilities for all cases are:

$$
\left\{\begin{array}{l}
P_{I}=(1-\tau)^{n} \\
P_{S_{-} B l k}=P_{S_{-} H O B}\left(\sum_{i=0}^{R^{*}} p_{d^{*}}^{i}\left(1-p_{d}^{*}\right)\right) \\
P_{C}=1-(1-\tau)^{n}-n \tau(1-\tau)^{n-1} \\
P_{D_{-} A C K}=n \tau(1-\tau)^{n-1} p_{d} \\
P_{D_{-} B A}=P_{S_{-} H O B} p_{d^{*}}^{R^{*}+1}
\end{array}\right.
$$

As throughput is defined by the expression in Eq. 9, the average successful payload size and average time of a full transmission cycle are required. If the size of payload is fixed as $L_{P l d}$, the number of MPDUs in one block is $N$, then the average successful payload size is $E\left[L_{P l d}\right]=P_{S_{-} H O B} L_{P l d}+$ $P_{S} B l k(N-1) L_{P l d}$ then the average time duration is the sum of the product of time duration and state probability for each case. The throughput of Block ACK mechanism is therefore given by:

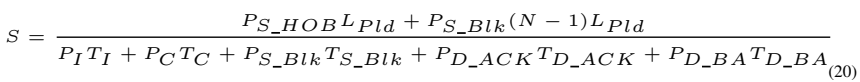

\section{NumericAl Results AND Discussion}

In this section, we present some numerical results of throughput performance for three different ARQ mechanisms implemented on a remote cloud-based SDR: basic DCF, Block ACK with basic access protection mechanism and Block ACK without protection mechanism. These results are based on the proposed analytical model with a realistic network latency distribution. First, performance of the three mechanisms are presented with introduced latency on the cloud-transceiver transmission network represented by a shifted Gamma distribution. Second, the throughput performance of different mechanisms are compared and discussed with changing parameters in shifted Gamma distribution. Third, fibre constraints are discussed for all three mechanisms.

For simplicity, a number of assumptions are made: the wireless channel is assumed to be error-free; there is no packet loss on the fibre links; and the data length for all MPDUs is constant and equal to the maximum 802.11 payload size (2312 bytes). The number of frames in each block is fixed at 20 , and the data rate is set to $150 \mathrm{Mbps}$. ACK_timeout and BA_timeout are $314 \mu s$ and $628 \mu s$ respectively. For realistic modelling, retransmission of BAR is considered when BAR is not acknowledged within the timeout period.

\section{A. Performance of Different Mechanisms}

The mean $(\mu)$ and variance $(\sigma)$ of the delay are $10 \mathrm{~ms}$ and $400 \mathrm{~ms}^{2}$ respectively. The minimum propagation delay $(\delta)$ is set to $100 \mu \mathrm{s}$. Due the property of shifted Gamma distribution, the shape parameter $k=(\mu-\delta)^{2} / \sigma$; and scale parameter $\theta=\sigma /(\mu-\delta)$, so in this case $k=0.245$ and $\theta=40.40$. The late ACK probability in this distribution is 0.695 . Throughput performance with this specific parameter set is presented in Fig. 3.

Block ACK with protection mechanism performs best across any number of STAs under these conditions. Without the protective mechanism, the throughput performance of Block

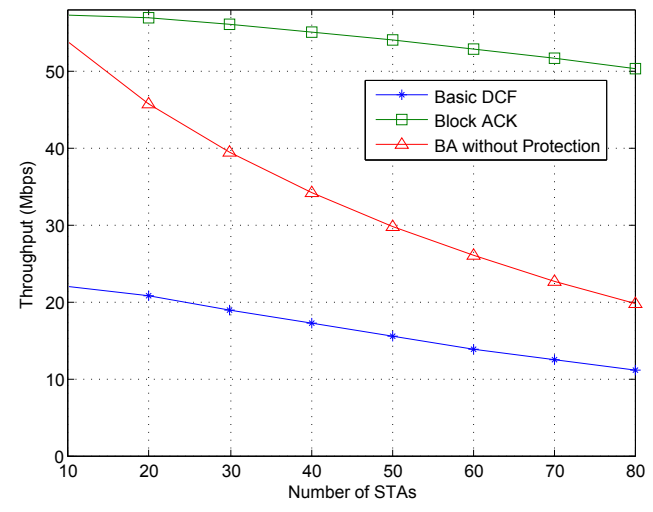

Fig. 3. Throughput as a function of the number of contending STAs

ACK is heavily affected by the number of STAs $(n)$ : the throughput difference between Block ACK with basic DCF access mechanism is only $17.7 \mathrm{Mbps}$ when $n=10$ while the difference increases to 41.9 Mbps when $n=80$. Due to the overhead of ACK transmission, basic DCF provides the lowest throughput of the three mechanisms. The throughput of basic DCF decreases almost linearly as $n$ increases. When $n=80$, the throughput performance of basic DCF and Block ACK without protective mechanism are very similar.

\section{B. Performance Under Various Network Conditions}

In this set of tests, in order to model different network situations, we utilise different values of variance $(\sigma)$ in the shifted Gamma distribution, while the mean $(\mu)$ remains set to $5 \mathrm{~ms}$. The number of nodes in the test system is 10 and the minimum propagation delay is $100 \mu \mathrm{s}$.

As shown in Fig. 4, the throughput performance for all three mechanisms improves as $\sigma$ increases. This is because the mean of network latency is normally larger than ACK_timeout and BA_timeout, so when the variance in latency is small, the probability of late ACKs is very high. Additionally, the gamma distribution is an asymmetric distribution; values smaller than mode/mean always have higher probability than values greater than mode. So when variance is high, the likelihood that latency will be less than the mode/mean is higher than the likelyhood of latency greater than the mode/mean.

BA without protection archives highest performance before the variance reaches $50 \mathrm{~ms}^{2}$. This is because BA without protection mechanism does not need to wait for the ACK to return, hence the only determinant of success or failure is the return of BA and that BA_timeout has higher tolerance than $A C K_{-}$timeout. However, the limitation of higher collision probability limits the performance of BA without protection. So when variance is higher, the network situation gives a higher probability for immediate ACK return before the timeout expires, and BA with basic DCF performs best. Basic DCF with smallest throughput performance wastes much of the bandwidth in the process of waiting for ACKs.

\section{Increasing Fibre Length Support}

This set of tests contains 10 nodes with mean of $10 \mu \mathrm{s}$ and variance of $400 \mathrm{~ms}^{2}$. When the fibre length is increased 


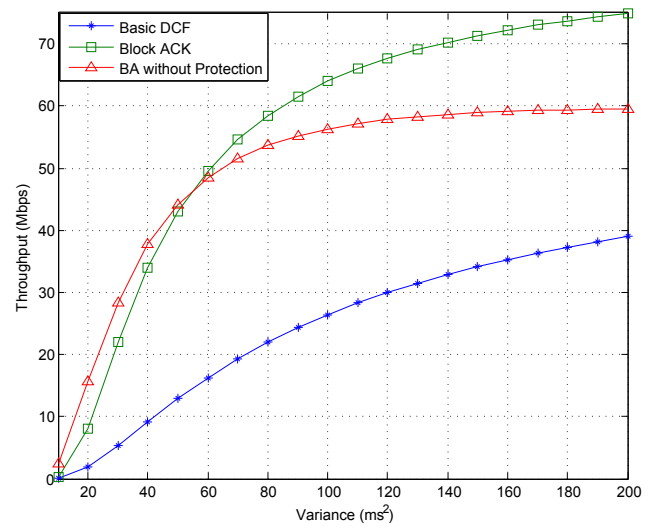

Fig. 4. Throughput as a function of variance of the shifted gamma distribution

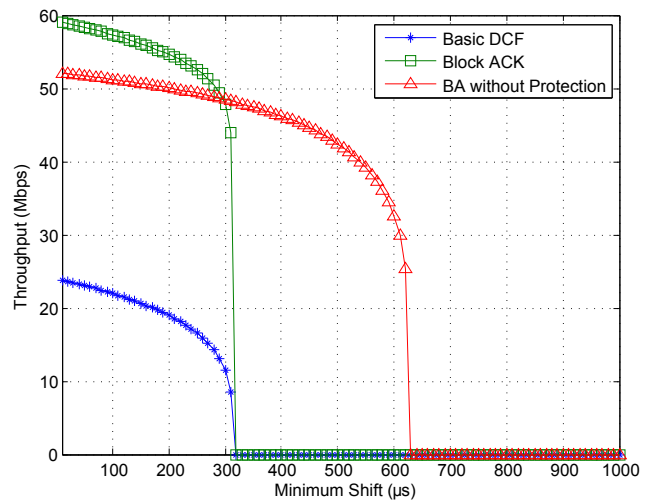

Fig. 5. Throughput as a function of minimum propagation delay

further, throughput will be reduced due to the extra introduced latency. When the one-way propagation delay exceeds half of either the ACK timeout or the Block ACK timeout, the transmitter will never receive the feedback of ACK or BA within the timeout period regardless of how many retransmission attempts are made. Therefore, the throughput drops to zero when the one-way propagation delay on the fibre link reaches the value of half of the ACK timeout. This means that the ACK timeout value constrains the fibre transmission range when the basic DCF mechanism is used. Additionally, when the protective mechanism of Block ACK is HOB (which also uses legacy DCF) for the first frame in the block, such cliff-like behaviour is also exhibited. However, the Block ACK without protective mechanism supports a larger maximum value because the timeout value for BAR is much longer than ACK as shown in Fig. 5.

\section{CONCLUSION}

In this paper, an enhanced analytical model for the throughput of IEEE 802.11 implementation on cloud-like networks using RoF technology has been developed. A variety of results are presented to illustrate the impact of the statistical properties of the cloud-transceiver transport network on legacy DCF, Block ACK with protective mechanism and Block ACK without protective mechanism. It is demonstrated that Block ACK can enhance protocol performance when the probability of late acknowledgement is high; however, performance is still constrained, as with legacy DCF, by the minimum propagation latency of optical fibre. Block ACK without protective mechanism provides smaller improvements than when the protection mechanism is enabled, but it supports longer optical fibre links due to the longer Block ACK timeout value.

\section{REFERENCES}

[1] J. Segal and M. Weldon, "lightradio portfolio - technical overview technology white paper \#1," Alcatel-Lucent White Paper, Jun 2011.

[2] Y. Lin, L. Shao, Z. Zhu, Q. Wang, and R. K. Sabhikhi, "Wireless network cloud: Architecture and system requirements," IBM Journal of Research and Development, vol. 54, no. 1, pp. 4:1 -4:12, januaryfebruary 2010.

[3] A. Ng'oma, "Radio-over-fibre technology for broadband wireless communication systems," Ph.D. dissertation, 2005.

[4] H. B. Kim, "Radio over fiber based network architecture," Ph.D. dissertation, Universitätsbibliothek, 2005.

[5] Y. Xiao and J. Rosdahl, "Performance analysis and enhancement for the current and future IEEE 802.11 MAC protocols," SIGMOBILE Mob. Comput. Commun. Rev., vol. 7, no. 2, p. 619, Apr. 2003. [Online]. Available: http://doi.acm.org/10.1145/950391.950396

[6] B. Kalantari-Sabet, M. Mjeku, N. Gomes, and J. Mitchell, "Performance impairments in single-mode radio-over-fiber systems due to MAC constraints," Journal of Lightwave Technology, vol. 26, no. 15, pp. 2540-2548, 2008.

[7] S. Deronne, V. Moeyaert, and S. Bette, "Analysis of the MAC performances in $802.11 \mathrm{~g}$ radio-over-fiber systems," in 18th IEEE Symposium on Communications and Vehicular Technology in the Benelux (SCVT), Ghent, Belgium, Nov. 2011, pp. 1-5.

[8] — "Impact of the slottime parameter value on the MAC performances in IEEE 802.11 wireless systems using radio-over-fiber technology," in 17th IEEE Symposium on Communications and Vehicular Technology in the Benelux (SCVT), Enschede, Netherlands, Nov. 2010, pp. 1-6.

[9] _ "WiFi transmission in radio-over-fiber systems: Performance of the IEEE 802.11n aggregation mechanism," in International Conference on Optical Network Design and Modeling (ONDM), Brest, France, Apr. 2013, pp. 167-172.

[10] Y. Lin, L. Shao, Z. Zhu, Q. Wang, and R. K. Sabhikhi, "Wireless network cloud: Architecture and system requirements," IBM Journal of Research and Development, vol. 54, no. 1, pp. 4:1-4:12, 2010.

[11] I. Gomez, V. Marojevic, and A. Gelonch, "Aloe: An open-source SDR execution environment with cognitive computing resource management capabilities," IEEE Communications Magazine, vol. 49, no. 9, pp. 76$83,2011$.

[12] N. Ghazisaidi and M. Maier, "Fiber-wireless (FiWi) access networks: Challenges and opportunities," IEEE Network, vol. 25, no. 1, pp. 36-42, 2011.

[13] H. Li and L. Mason, "Estimation and simulation of network delay traces for VoIP in service overlay network," in International Symposium on Signals, Systems and Electronics, Montreal, Quebec, Canada, Jul. 2007, pp. 423-425.

[14] "IEEE std 802.11 - 2007 (Revision of IEEE 802.11 - 1999) part 11: Wireless LAN medium access control (MAC) and physical layer (PHY) specifications," 2007.

[15] G. Bianchi, "Performance analysis of the IEEE 802.11 distributed coordination function," IEEE Journal on Selected Areas in Communications, vol. 18 , no. 3, pp. 535-547, March 2000.

[16] H. Wu, Y. Peng, K. Long, S. Cheng, and J. Ma, "Performance of reliable transport protocol over IEEE 802.11 wireless LAN: analysis and enhancement," in IEEE INFOCOM, Annual Joint Conference of the IEEE Computer and Communications Societies, vol. 2, New York, USA, Jun. 2002, pp. 599-607.

[17] Q. Ni, T. Li, T. Turletti, and Y. Xiao, "Saturation throughput analysis of error-prone 802.11 wireless networks," Wireless Communications and Mobile Computing, vol. 5, no. 8, pp. 945-956, 2005. [Online]. Available: http://onlinelibrary.wiley.com/doi/10.1002/wcm.358/abstract 